\title{
Locked Nucleic Acid: Tighter is Different
}

\author{
Jonathan K. Watts* \\ Received (in $X X X, X X X)$ Xth $X X X X X X X X X 20 X X$, Accepted Xth XXXXXXXXX 20XX \\ DOI: 10.1039/c3cc40340h
}

\begin{abstract}
5 This viewpoint briefly reviews the impact of Locked Nucleic Acid (LNA) oligonucleotides, first described in a ChemComm paper in 1998. A number of unique applications in oligonucleotide biotechnology have been made possible by the high binding affinity and specificity of LNA, and these ${ }_{10}$ provide the main focus of the viewpoint.
\end{abstract}

I recently read a fascinating analysis of the role of the internet in the uprisings in Egypt, Tunisia and elsewhere over the past several years. Did social media really change anything fundamental that allowed these uprisings, or did they simply

15 make communication faster? Zeynep Tufecki's response is that faster is different: the speed of person-to-person communication afforded by the internet allowed coordination of protests and circulation of ideas in ways that simply wouldn't have been possible at all with older types of communication (e.g. state${ }_{20}$ controlled mass media or one-on-one conversations). ${ }^{1}$

A parallel question could be posed about the effect of locked nucleic acid (LNA, Figure 1) on oligonucleotide chemical biology. Has it really changed the field, or does it simply bind with higher affinity than its predecessors? And I would contend 25 that the answer is again parallel: tighter binding is different. In this viewpoint I will examine several developments in oligonucleotide biotechnology that were made possible by LNA's very high binding affinity and specificity.

Foundational papers on LNA were independently published by 30 both the Wengel ${ }^{2,3}$ and Imanishi ${ }^{5}$ groups. Wengel's ChemComm paper, ${ }^{2}$ the subject of this viewpoint, was the first publication of LNA oligonucleotides and their dramatically increased binding affinity. For mixed sequences, inserting several locked nucleotides into a DNA oligomer gave a stabilization of about ${ }_{35} 5{ }^{\circ} \mathrm{C}$ per locked nucleotide when binding to DNA and $7-8{ }^{\circ} \mathrm{C}$ when binding to RNA. ${ }^{2}$ Moreover, the binding showed high specificity in that a single mismatched base opposite LNA led to a more pronounced drop in denaturation temperature than a mismatch opposite DNA. ${ }^{9}$

40 Nucleic acid duplex formation is generally driven by enthalpy, and disfavoured by entropy. Before the development of LNA, there was already an awareness that preorganization might be able to reduce the entropic penalty of duplex formation. ${ }^{10}$ And if the preorganized conformation was appropriate for binding, the 45 enthalpic term would be maintained, thus increasing the overall binding affinity. Other ways to increase the strength of duplex binding, including addition of positive charge and conjugation of intercalators or minor-groove binders, can decrease the specificity

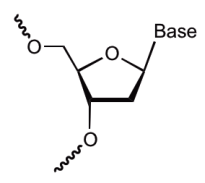

DNA

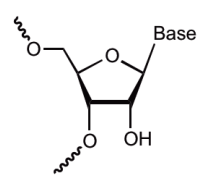

RNA

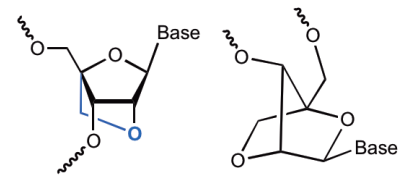

LNA
Figure 1. A comparison of the structures of DNA, RNA and LNA. LNA is shown both from the normal perspective of drawing nucleotides and the normal perspective of drawing [2.2.1] bicyclic structures.

of binding, while preorganization showed the potential to 50 increase both affinity and specificity. ${ }^{10}$

Thus a number of groups had been working on constrained nucleotides for several years before LNA was published. None of the previous constrained analogues, however, showed the degree of increase in binding affinity conferred by LNA. It is 55 interesting that LNA, the most successful of the constrained nucleic acids, is also arguably the simplest conceivable structure, bearing only a single extra atom relative to RNA (Figure 1).

The oligonucleotide community, therefore, warmly welcomed the arrival of LNA. The compatibility of LNA synthesis with 60 normal phosphoramidite coupling chemistry allowed it to be applied very readily to the full range of challenges facing the field.

LNA was very quickly applied to the field of gene silencing ${ }^{11}$ by antisense oligonucleotides (ASOs, Figure 2). LNA-modified ${ }_{65}$ ASOs often show increased potency relative to other chemically modified ASOs. ${ }^{12,13}$ Five LNA oligonucleotides are in clinical trials. $^{14}$

More recently, the high binding affinity of LNA was applied to allow the development of much shorter ASOs than previously 70 thought possible. ${ }^{4,15}$ Sequences as short as 12 -mers, containing LNA wings surrounding an 8-mer DNA window, were active in huh-7 cells and in vivo in mice and monkeys. ${ }^{4}$ Longer oligonucleotides up to 15 or 16-mers showed similar potencies in cell culture $\left(\mathrm{IC}_{50}\right.$ values of $\sim 0.5 \mathrm{nM}$ ) but lower efficacies in vivo. 75 Some subsequent studies have found that 16-mers may be preferable to 12-mers for certain applications, ${ }^{16}$ but even $16 \mathrm{nt}$ is shorter than would have previously been considered for most antisense chemistries. Short LNA-modified ASOs are also ideal for delivery to cultured cells in the absence of lipid, termed ${ }_{80}$ gymnotic delivery. $^{17}$

Certain LNAs have significant toxicity, ${ }^{13}$ but it is generally possible to find sequences with an acceptable toxicity profile. Nevertheless, an antisense oligonucleotide targeting PCSK9 was recently pulled from clinical trials after acute kidney injury was 
observed in one patient in a Phase I trial. ${ }^{18}$ It is not clear how much of this toxicity was due to unintended but on-target gene silencing in the kidney, and how much to sequence-dependent off-target effects. Nor is it clear how much toxicity was due to 5 LNA, and how much to other aspects of the oligonucleotide chemistry (see below for a discussion of backbone toxicity). Nevertheless, this failed trial is a setback for LNA and for the antisense approach.

Another gene-silencing approach, RNA interference, can also 10 benefit from LNA oligonucleotides. ${ }^{19}$ The RNAi technique
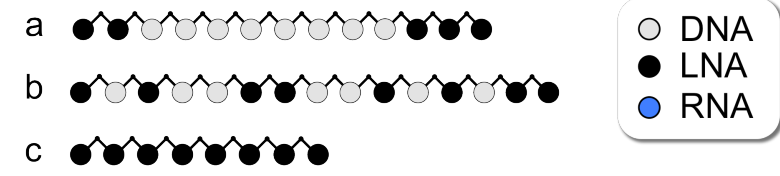

\section{d oroborosoros oroboroborosos

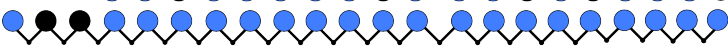

Figure 2. Gene silencing approaches and LNA, showing designs from the listed references in schematic form. (a) Gapmer ASOs contain high-affinity modifications on the wings and DNA in the middle, allowing their target to be cleaved by RNase H. Potent LNA gapmers can be as small as $12-13$ mers. ${ }^{4}$ (b) Mixmer LNAs can be used as steric blocking oligonucleotides - e.g. to inhibit miRNAs, inhibit translation or redirect splicing. ${ }^{6}$ (c) Fully modified 8-mer "tiny LNAs" can bind the seed sequence shared between a family of miRNAs. ${ }^{7}$ (d) small internally segmented interfering RNAs (sisiRNAs) contain a nick in the passenger strand but are taken up by the RNAi machinery. ${ }^{8}$

requires double-stranded small interfering RNA (siRNA), and one concern is that off-target effects may arise from the so-called "passenger strand." To avoid this problem, a variant siRNA design was used where the passenger strand was internally 15 segmented, giving two short strands rather than one long strand. ${ }^{8}$ This would not have been possible without the high binding affinity of LNA (Figure 2).

High binding affinity has proven particularly important in the rapidly expanding field of microRNA (miRNA) research. 20 miRNAs are 20 -nucleotide sequences of RNA with important biological roles. ${ }^{20}$ Because of their small size, inhibition and analysis of miRNAs is challenging with normal oligonucleotides.

Inhibition of miRNAs in cells and in vivo is a key approach to study miRNA function and can induce a therapeutic effect. As 25 for traditional mRNA-targeted antisense oligonucleotides, chemical modification is key to effective anti-miRNA ASO activity. A 15-mer LNA mixmer oligonucleotide that targets miR-122 was effective in non-human primates and is currently in Phase II clinical trials for treatment of hepatitis C (Figure 2b). ${ }^{6}$

30 The high binding affinity of LNA allows it to go a step further than traditional miRNA inhibition, however. Using a fullymodified 8-mer "tiny LNA" (Figure 2c), it is possible to inhibit a family of miRNAs with a common seed sequence (the 6-8 nucleotides at the 5 '-end of the active miRNA). ${ }^{7}$ The seed ${ }_{35}$ sequence is key for recognition of target RNAs, ${ }^{20}$ so this approach targets a group of miRNAs that share a common inhibitory profile. The tiny LNAs did not appear to be toxic or to downregulate mRNAs containing perfectly complementary sites. ${ }^{7}$

Beyond targeting various classes of RNA, another therapeutic 40 gene silencing approach could involve targeting of chromosomal DNA. LNA can be used as a triplex-forming oligonucleotide at
dsDNA, and intriguingly it has also been used to strand-invade and bind DNA through Watson-Crick base pairing. ${ }^{21,22}$ This property had previously only been seen for neutral-backbone 45 analogues like peptide nucleic acid. The direct binding to DNA was confirmed by a biotin-based chromatin immunoprecipitation experiment. $^{21}$

LNA has had an impact in the field of diagnostics and bioanalysis as well. For example, designing a DNA microarray $5_{0}$ to detect levels of different miRNAs from a tumour sample would be challenging because the DNA probes would have different
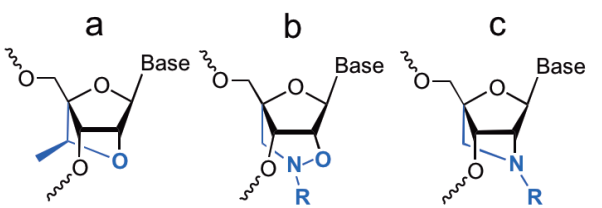

d

(S)-CET

$$
\begin{array}{ll}
\text { 2',4'-BNA }^{\text {NC }} & \text { 2'-amino LNA }^{\prime} \text { - }- \text {-L-LNA } \\
\mathbf{R}=\mathrm{H}, \mathrm{Me} & \begin{array}{l}
\mathrm{R}=\mathrm{CH}_{2} \text {-pyrene } \\
\text { or other groups }
\end{array}
\end{array}
$$

Figure 3. Several next-generation bridged nucleic acids.

melting temperatures depending on the sequence of the miRNA. This means that for a given annealing temperature, some of the DNA probes would bind more tightly to their target than others, 55 making normalization very difficult. LNA-modified arrays can be designed to have a uniform and high melting temperature for all probes, increasing the sensitivity of the array and giving consistent quantitation across the full range of miRNAs. ${ }^{23}$

Quantitative real-time PCR is another diagnostic approach that ${ }_{60}$ can be improved by LNA technology. In 5'-nuclease assay PCR, a fluorescent probe oligonucleotide is degraded by the Taq polymerase. This probe should have a higher binding affinity than the PCR primers used. Yet in certain applications the probe should also be short, and must bind with high specificity, to ${ }_{65}$ detect single-nucleotide mismatches. LNA is an ideal candidate with this range of properties and has been used to develop probes with higher sensitivity and specificity. ${ }^{24}$

The high binding affinity of LNA, of course, also comes with inherent challenges. LNA oligonucleotides can form stable self70 structures (homoduplexes or hairpins), which are particularly stable if they include LNA-LNA base pairs. This is one of the main reasons why oligonucleotides $>8 \mathrm{nt}$ should generally not be fully modified with LNA. Design of gapmer or mixmer oligonucleotides (including the placement of LNA modifications) 75 must take into account the potential for stable self-structure.

As with most successes, LNA has inspired a family of derivatives. These are too numerous to detail here with the exception of several highlights (Figure 3). One LNA-inspired modification of particular note is the "constrained ethyl" or $(S)$ 80 cEt chemistry developed by ISIS and which is now, like LNA, in clinical trials (Figure 3a). cEt modification confers high binding affinity like LNA, and in some cases may show reduced toxicity. ${ }^{15}$ Another "next-generation" bridging moiety is known as 2',4'-BNA ${ }^{\mathrm{NC}}$ (Figure 3b), which shows increased selectivity for 85 binding RNA over single-stranded DNA, and increased triplexforming ability (binding to duplex DNA). ${ }^{25}$

A different type of LNA-based innovation is the use of nitrogen rather than oxygen as the bridging heteroatom. This 2'- 
amino analogue of LNA has allowed functional pendant groups to be projected into the minor groove of the duplex (Figure $3 \mathrm{c}$ ). ${ }^{26}$

Finally it should be noted that the full family of eight LNArelated stereoisomers has been tested and remarkably, six of them 5 bind RNA with high affinity. ${ }^{27}$ Among these, $\alpha$-L-LNA shows considerable promise (Figure 3d). In contrast to LNA, which induces strongly A-form helices, $\alpha$-L-LNA leads to a more Bform helical structure. ${ }^{28}$ As such, it is more of a DNA mimic than the parent compound, but retains the rigidity of LNA (in 10 contrast to DNA itself which is inherently quite flexible).

LNA has had a major impact across the spectrum of oligonucleotide biotechnology. However, there are a number of challenges in using oligonucleotides that LNA has not addressed. 15 The preeminent example is the challenge of delivery. Conjugation and formulation strategies are making great progress, but there is still a lot of work to be done, particularly for the question of cell-type-specific delivery. The development of clinically validated strategies to target tissues outside of the liver, 20 vasculature and kidneys - ideally without significant exposure to these organs - would open new doors for oligonucleotide therapeutics in general.

Secondly, for therapeutic purposes LNA is generally used in combination with a phosphorothioate (PS) backbone. In this way 25 it is like other antisense oligonucleotides in the clinic today. The PS backbone provides outstanding nuclease stability and helps with the delivery problem. However, PS gapmers tend to have high toxicity. Mipomersen, a phosphorothioate MOE gapmer approved by the FDA in early 2013, is associated with a risk of 30 hepatotoxicity and is only available through a careful risk evaluation and mitigation program. Thus in the areas of both sugar and phosphate modification, it is essential that chemists continue to develop creative ways to continue to improve potencies and toxicity profiles.

In this brief viewpoint I have highlighted a few areas in which the high binding affinity of LNA has allowed totally novel approaches - areas in which "tighter" has led to "different." These include short antisense oligonucleotides, "tiny LNAs" for 40 inhibition of miRNA families, three-stranded siRNAs, strand invasion of dsDNA at promoters, optimized miRNA arrays and short, specific probes for real-time PCR. Many of these approaches have been demonstrated only in the past few years. LNA research continues to be a fruitful area and further creative 45 applications will surely be explored in the years to come.

\section{Notes and references}

Department of Chemistry and Institute for Life Sciences, University of Southampton, SO17 1BJ, UK. Tel: +44 (0)23 8059 7679; E-mail: j.k.watts@soton.ac.uk

1. The full blog post is available on Zeynep Tufecki's "Technosociology" blog, http://technosociology.org/?p=424 (Date of post is 13 April 2011; most recently accessed 29 April 2013).

2. S. K. Singh, P. Nielsen, A. A. Koshkin and J. Wengel, Chem. ${ }_{55}$ Commun., 1998, 455-456.

3. A. A. Koshkin, S. K. Singh, P. Nielsen, V. K. Rajwanshi, R. Kumar, M. Meldgaard, C. E. Olsen and J. Wengel, Tetrahedron, 1998, 54, $3607-$ 3630 .
4. E. M. Straarup, N. Fisker, M. Hedtjarn, M. W. Lindholm, C. 60 Rosenbohm, V. Aarup, H. F. Hansen, H. Orum, J. B. Hansen and T. Koch, Nucleic Acids Res, 2010, 38, 7100-7111.

5. S. Obika, D. Nanbu, Y. Hari, K. Morio, Y. In, T. Ishida and T. Imanishi, Tetrahedron Lett., 1997, 38, 8735-8738; S. Obika, D. Nanbu,

Y. Hari, J.-i. Andoh, K.-i. Morio, T. Doi and T. Imanishi, Tetrahedron 65 Lett., 1998, 39, 5401-5404.

6. R. E. Lanford, E. S. Hildebrandt-Eriksen, A. Petri, R. Persson, M. Lindow, M. E. Munk, S. Kauppinen and H. Orum, Science, 2010, 327, 198-201.

7. S. Obad, C. O. Dos Santos, A. Petri, M. Heidenblad, O. Broom, C. 70 Ruse, C. Fu, M. Lindow, J. Stenvang, E. M. Straarup, H. F. Hansen, T. Koch, D. Pappin, G. J. Hannon and S. Kauppinen, Nat. Genet., 2011, 43, 371-378.

8. J. B. Bramsen, M. B. Laursen, C. K. Damgaard, S. W. Lena, B. Ravindra Babu, J. Wengel and J. Kjems, Nucleic Acids Res., 2007, 35, 75 5886-5897.

9. The improved specificity of binding was more definitively established in subsequent papers, including ref 3.

10. E. T. Kool, Chem. Rev., 1997, 97, 1473-1487.

11. J. K. Watts and D. R. Corey, J. Pathol., 2012, 226, 365-379.

80 12. The first major study on LNA-modified ASOs was published in 2000 and has been cited over 300 times since. See, C. Wahlestedt, P. Salmi, L. Good, J. Kela, T. Johnsson, T. Hokfelt, C. Broberger, F. Porreca, J. Lai, K. Ren, M. Ossipov, A. Koshkin, N. Jakobsen, J. Skouv, H. Oerum, M. H. Jacobsen and J. Wengel, Proc. Natl. Acad. Sci. U. S. A., 2000, 97, 85 5633-5638.

13. E. E. Swayze, A. M. Siwkowski, E. V. Wancewicz, M. T. Migawa, T. K. Wyrzykiewicz, G. Hung, B. P. Monia and C. F. Bennett, Nucleic Acids Res, 2007, 35, 687-700.

14. Details of these compounds, targets and trials can be found on the 90 website of Santaris Pharma A/S, http://www.santaris.com

15. P. P. Seth, A. Siwkowski, C. R. Allerson, G. Vasquez, S. Lee, T. P. Prakash, E. V. Wancewicz, D. Witchell and E. E. Swayze, J. Med. Chem., 2009, 52, 10-13.

16. F. Modarresi, M. A. Faghihi, M. A. Lopez-Toledano, R. P. Fatemi, 95 M. Magistri, S. P. Brothers, M. P. van der Brug and C. Wahlestedt, Nat. Biotechnol., 2012, 30, 453-459.

17. C. A. Stein, J. B. Hansen, J. Lai, S. Wu, A. Voskresenskiy, A. Hog, J. Worm, M. Hedtjarn, N. Souleimanian, P. Miller, H. S. Soifer, D. Castanotto, L. Benimetskaya, H. Orum and T. Koch, Nucleic Acids Res, $1002010, \mathbf{3 8}$, e3.

18. E. P. van Poelgeest, R. M. Swart, M. G. Betjes, M. Moerland, J. J. Weening, Y. Tessier, M. R. Hodges, A. A. Levin and J. Burggraaf, Am. J. Kidney Dis., 2013, in press, DOI: 10.1053/j.ajkd.2013.1002.1359.

19. J. Elmen, H. Thonberg, K. Ljungberg, M. Frieden, M. Westergaard, $105 \mathrm{Y}$. Xu, B. Wahren, Z. Liang, H. Urum, T. Koch and C. Wahlestedt, Nucleic Acids Res., 2005, 33, 439-447.

20. D. P. Bartel, Cell, 2009, 136, 215-233

21. R. Beane, S. Gabillet, C. Montaillier, K. Arar and D. R. Corey, Biochemistry, 2008, 47, 13147-13149.

110 22. E. M. Zaghloul, A. S. Madsen, P. M. Moreno, Oprea, II, S. ElAndaloussi, B. Bestas, P. Gupta, E. B. Pedersen, K. E. Lundin, J. Wengel and C. I. Smith, Nucleic Acids Res, 2011, 39, 1142-1154.

23. M. Castoldi, S. Schmidt, V. Benes, M. Noerholm, A. E. Kulozik, M. W. Hentze and M. U. Muckenthaler, $R N A$, 2006, 12, 913-920.

115 24. E. Reynisson, M. H. Josefsen, M. Krause and J. Hoorfar, J. Microbiol. Methods, 2006, 66, 206-216; S. Salvi, F. D'Orso and G. Morelli, J. Agric. Food. Chem., 2008, 56, 4320-4327.

25. S. M. A. Rahman, S. Seki, S. Obika, H. Yoshikawa, K. Miyashita and T. Imanishi, J Am Chem Soc, 2008, 130, 4886-4896.

120 26. A number of different pendant groups and linkers have been tested. For one excellent example of this approach see, P. J. Hrdlicka, B. R. Babu, M. D. Sorensen and J. Wengel, Chem Commun, 2004, 1478-1479. 27. V. K. Rajwanshi, A. E. Hakansson, M. D. Sorensen, S. Pitsch, S. K. Singh, R. Kumar, P. Nielsen and J. Wengel, Angew Chem Int Ed Engl, 125 2000, 39, 1656-1659.

28. J. T. Nielsen, P. C. Stein and M. Petersen, Nucleic Acids Res., 2003, 31, 5858-5867. 